TITLE:

\title{
Whom to Follow on social trading services? A system to support discovering expert traders
}

$\operatorname{AUTHOR}(S)$ :

Lee, Woonyeol; Ma, Qiang

\section{CITATION:}

Lee, Woonyeol ... [et al]. Whom to Follow on social trading services? A system to support discovering expert traders. 2015 Tenth International Conference on Digital Information Management (ICDIM) 2016: 188-193

\section{ISSUE DATE:}

2016-01-13

URL:

http://hdl.handle.net/2433/217602

\section{RIGHT:}

(C) 2015 IEEE. Personal use of this material is permitted. Permission from IEEE must be obtained for all other uses, in any current or future media, including reprinting/republishing this material for advertising or promotional purposes,

creating new collective works, for resale or redistribution to servers or lists, or reuse of any copyrighted component of this work in other works.; This is not the published version. Please cite only the published version.; この論文は出版社版

でありません。引用の際には出版社版をご確認ご利用ください。 


\section{Whom to Follow on Social Trading Services? A System to Support Discovering Expert Traders}

\author{
Woonyeol Lee \\ Graduate School of Informatics \\ Kyoto University, Kyoto, Japan 606-8501 \\ Email: unyollee@db.soc.i.kyoto-u.ac.jp
}

\author{
Qiang Ma \\ Graduate School of Informatics \\ Kyoto University, Kyoto, Japan 606-8501 \\ Email: qiang@i.kyoto-u.ac.jp
}

\begin{abstract}
Social trading services, the financial services connected with social networking services are in the spotlight. With social trading services, users can follow and automatically imitate expert traders' trades. Finding expert traders who have good, consistent performance to follow is one of the key challenges. To address this issue, we propose a system we call W2F (Whom to Follow) to help users discover expert traders. By analyzing a trader's trading history and comparing it with that of other traders, W2F estimates traders with three measures: performance, risk, and consistency. The results are visualized to help users make decisions on whom to follow. We describe experiments we carried out to validate these three measures and demonstrate the usefulness of W2F on the basis of a user study.
\end{abstract}

\section{INTRODUCTION}

Social trading is a new investment way in which users (traders) can share opinions and investment information with others. Social trading services such as Zulutrade [10], Ayondo [11], and eToro [12] aim to open communication with various main agents that goes beyond the type of communication that can be achieved with limited information between closed investment communities. Social trading mainly for foreign exchange can also be applied to various financial investments such as stock, real estate, and bond trading [8]. The practical effect of social trading is positive and achieves higher ROI (Return On Investment) than any other trading methods [2].

Social Trading is a new investment paradigm that is totally different from traditional investment methods. Traditional investment methods try to discover and analyze proper prices to find out essential values of things on the basis of demand and supply, the market's basic principle. In other words, traders should study the market and use it as a basis for considering their portfolio composition. In contrast, sharing investment strategy is the key feature of social trading. Users can learn and imitate experts' trading and investment methods with such sharing. The function that users mainly use is following expert traders and automatically imitating their trades instead of themselves making a decision for each trade. In other words, social trading services provide a people-based portfolio composition function rather than the traditional market-based one. Therefore, one of the important challenges of utilizing social trading services is to make proper decisions about whom to follow, because profit depends on traders whom we are following.
A social trading service, such as Zulutrade [10], usually provides ranking functions for recommending expert traders to follow. Such ranks are presumed to be assigned on the basis of traders' performances with respect to past profit. Usually, the profit-based ranks change frequently because some traders carry out trades in a high-risk, high-return manner. It is therefore risky to follow traders by referring to profits only. Intuitively, an expert trader achieves consistently good performance with low risk. That is to say, we need to estimate traders by considering their levels of performance, risk, and consistency. Although social trading services also provide detailed information about traders such as profits and trading histories on the traders' profile pages, it is not easy to find expert traders to follow without a total understanding of the information because the pages do not provide explicit indicators about the performance, risk, and consistency of traders.

Accordingly, in this paper we propose a system we call W2F (Whom to Follow), to help users discover expert traders with three measures: performance, risk, and consistency. We analyze the performance and risk levels of traders by comparing their trading records with those of others. We also estimate their consistency by analyzing their behavior represented by the historical trade records. The idea is simple: a trader who consistently yields fairly returns with low risk is a considerably expert trader whom we can follow. These three measures can be used separately or in an integrated manner for ranking traders flexibly. Using our system, we estimate each trader and then visualize the results to help users quickly find expert traders on the basis of their intentions (investment policies, etc.). The major contributions of this paper are summarized as follows.

- We describe a W2F system we propose for automatically estimating traders to help users discover expert traders to follow (Section III).

- We present three measures (performance, risk, and consistency) we propose as a means to rank traders (Section IV). To the best of our knowledge, this is the first attempt to perform the task of estimating traders on social trading services. We provide a relative ranking function to discover expert traders by considering two kinds of context: other traders and the experts' own past 
trades (Section IV). We also show how we considered the relationship between news events and trades to estimate the traders' performance level.

- We describe experiments we conducted by using real datasets collected from Zulutrade to demonstrate W2F (Section V). We first show how we evaluated the three measures (performance, risk and consistency) by applying them to rank traders. Then, we show how we evaluated $\mathrm{W} 2 \mathrm{~F}$ on the basis of a user survey.

\section{RELATED WORK}

Discovering expert users is one of the important challenges of online services. Conventional methods are based on opinion mining, network analysis, and user behavior analysis [5] [3]. For example, El-korany proposed a method to recommend experts on an online community [5] such as Stackoverflow [13]. Online communities are mainly composed of ordinary users and experts. They utilize the rating feature of social network services to identify expert users: a user with high evaluations from others is a considerable expert. However, the current social trading services do not provide such a mechanism for traders to rate each other. Macdonald et al. proposed a method of searching for experts effectively [3]. With this method the profiles of candidates are analyzed and high grades are assigned to users whose profile quality is high. Unfortunately, such information is not available on current social trading services. To the best of our knowledge, our work is the first attempt to perform this task.

Pan et al. performed a study on eToro, which is another famous social trading service. They separate trades into three types: single trades, copy trades, and mirror trades [2]. These three types respectively denote normal trades made by the users themselves, those made by copying those of other traders, and those made by automatically imitating other traders. In our work, we focused on mirror trades, which is the most popular type of the three [6] on social trading services. Pan et al. revealed that the average ROI (Return of Investment) of mirror trades is positive and higher than those of single and copy trades. They concluded that social trading helps yield positive returns. They also compared simple ranks (based on performance) with social ranks (based on the number of followers). Their experimental results show that these two kinds of rankings do not correlate with further performance (profits). In other words, historical performance or social support (number of followers) alone is not good enough yet to help us find expert traders.

Liu et al. compared social trades with non-social trades [6] in carrying out a large scale empirical study. The interesting observations from their work include, 1) most of the trades were held for less than an hour and very few trades were held for a very long time (over one month), 2) the chance of winning in mirror trades having a one minute to one week duration is much higher than 50 percent, 3) the time for holding a losing position is generally higher than that for holding a winning position, and 4) in mirror trades, the disposition effect (investors tend to sell financial assets whose price has increased while keeping assets that have dropped in value) is small. We utilized these observations to design our method of estimating traders.

Bauwens et al. have studied how news events affect volatility in the euro/dollar foreign exchange market [1]. They manually classify news events into two categories: scheduled and unscheduled events. They also classify trade time into three time periods with respect to news announcements: pre-announcement, contemporaneous, and postannouncement. Then they analyze the volatility responding to a news announcement. Their results reveal that both scheduled and unscheduled news events increase volatility during the preannouncement period. That is to say, news often changes the trends in the foreign exchange market. In short, news is one of the important factors for foreign exchange trading. As a result, an expert trader continually pays attention to market news. We utilized this feature to help us find expert traders.

\section{OVERVIEW OF W2F}

In contrast to the profit based ranking provided by social trading services such as Zulutrade, our W2F system evaluates traders from the aspects of performance, risk, and consistency. It visualized the evaluation results and presents them to users to help them discover expert traders to follow.

The system structure is shown in Fig. 1. The system's crawler collects trading data of traders in Zulutrade for further analysis. It also gathers the news data from ForexFactory[14] which is a well-known financial news site. Notice that we collected news data of the past eight years. The trading and news data are stored by using relational databases. The performance analyzer, risk analyzer, and consistency analyzer estimate traders performance, risk, and consistency. Then, each analyzer standardizes traders' performance, risk, and consistency scores from 0 to 100 on the basis of z-score standardization for easier comparison. Finally, the analysis results will be visualized and presented to users to help them make a decision on selecting expert traders to follow.

As shown in Fig. 2, W2F visualizes and presents the estimation results in three ways.

- Area A: A 3D chart plotting traders performance, risk, and consistency indicators is shown here. Users can group and select expert traders intuitively on the basis of their investment policy. In this chart, a selected trader is shown in red and the corresponding data appears in area B and is highlighted in area $\mathrm{C}$.

- Area B: A chart is used to visualize a selected trader's levels of performance, risk, and consistency. A user can select such a trader in area A or B.

- Area C: All the traders are listed here. The traders' ID (tid), profit, performance $(\mathrm{P})$, risk (R), consistency (C), and two selection buttons are included. By clicking the tid, that traders profile page on Zulutrade will open in Area D for further investigation. Profit obtained from Zulutrade denotes the total profit of a trader. By clicking "Exclude", the trader will be removed from the candidate 


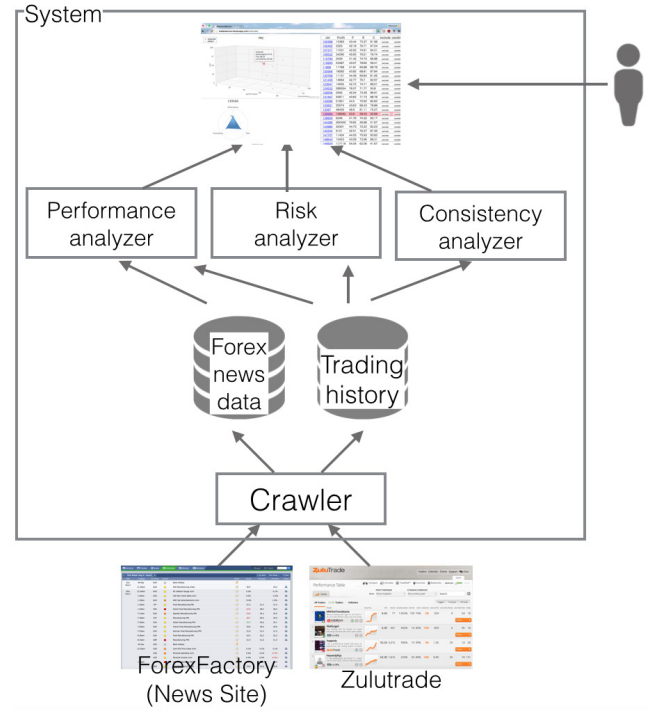

Fig. 1. Overview of W2F System

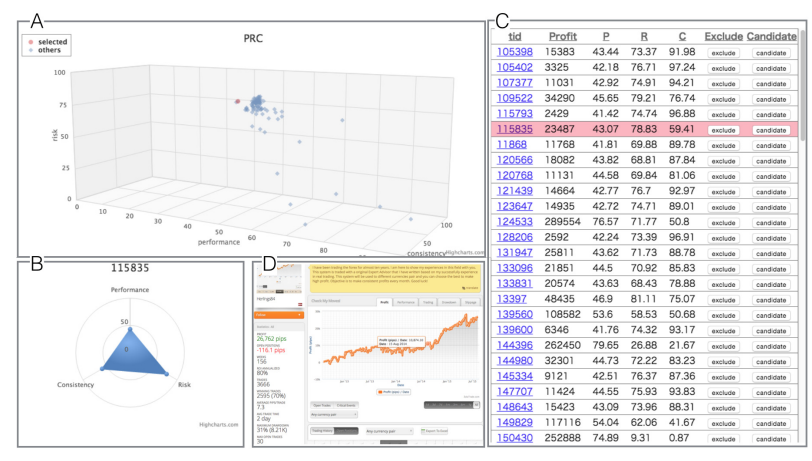

Fig. 2. Running Example of W2F: Presentation

list and the corresponding point in area A will be detected. On the other hand, clicking "Candidate"will keep the trader as a candidate expert for further analysis.

\section{Methodology}

As mentioned before, we propose three measures to estimate traders.

- Performance denotes how successful they have been in past trades. The assumption is made that high performance traders will make profits in the future. Currently, we use the profits and winning/losing ratios of traders to estimate their performance levels.

- Risk denotes the loss probability of trades. Currently, we use indicators such as drawdown and position opening time in trades to estimate the risk level of a trader.

- Consistency denotes the consistent level of a trader's trading patterns. For example, traders who perform trades in the same currency pairs and have a stable range of winning or losing money are said to perform consistently. We analyze the trading history to estimate the consistency level of a trader.

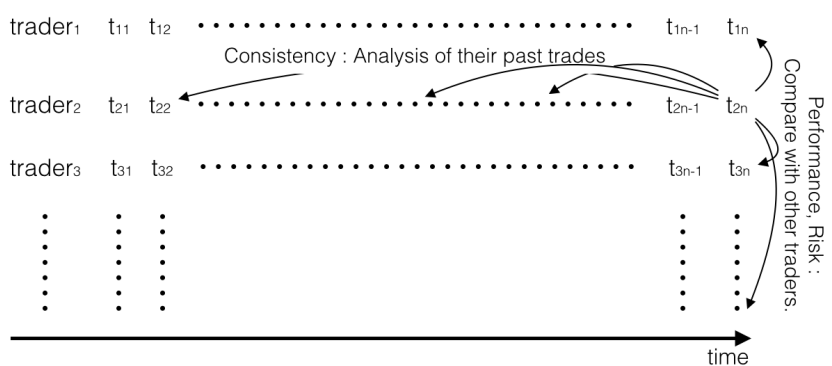

Fig. 3. Method Overview: 1) performance and risk estimation based on comparison analysis with other trades, 2) consistency analysis based on analysis of past trades.

Obviously, the levels of performance and risk are important measures to discover expert traders. However, due to the fact that the performance not only depends on the professional skill of a trader, but also on the market and other external conditions (policy, crisis, etc.), in contrast to the ranking mechanism provided by social trading services, we should estimate the performance and risk levels of traders in a relative manner.

As shown in Fig. 3, we propose methods of estimating performance and risk levels by comparing traders to each other. We also pay attention to the consistency of traders. As we mentioned before, we want to search for traders who yield profit consistently. This means an expert trader should not only have achieved a good past performance, but also has a high probability of making profits in the future. For this, we analyze traders' consistency by considering the past trades of each trader. The trading history of a trader is the second context used in our method. In short, we estimate traders' expertise from the three aforementioned aspects by comparing their trades with those of other traders and analyzing their trading history.

Actually, we can rank traders by using the above three measures solely or in combinational ways. Currently, our W2F system visualizes the estimation results and lets the users select expert traders by themselves. If users only pay attention to performance, they may choose the performance level as the ranking measure. Also, if a user wants to find expert traders with consistent high performances and low risk, an integrated ranking with performance, consistency, and risk is reasonable. We will discuss this issue in the section describing our experiments.

\section{A. Performance Estimation by Comparing with Other Traders}

We estimate the performance of a trader by using the factors shown in Table $\mathrm{I}^{1}$. Most of these factors can be obtained from the traders' profiles available on social trading services' Web sites.

As mentioned before, news announcements are important for trades [1]. Therefore, to estimate the performance level of a trader, we introduce factors related to news announcements:

\footnotetext{
${ }^{1}$ Notice that these factors (for estimation of performance, risk, and consistency) are selected on the basis of observations in a previous study introduced in Section II.
} 
TABLE I

FACTORS USED FOR PERFormance Estimation

\begin{tabular}{ll}
\hline \hline pip & $\begin{array}{l}\text { Price interest point: a pip measures the amount of change } \\
\text { in the exchange rate for a currency pair in a trade. }\end{array}$ \\
win_rate & $\begin{array}{l}\text { Percentage of trades making a positive profit among all } \\
\text { trades. }\end{array}$ \\
avg_pip & $\begin{array}{l}\text { Average pip of all trades. } \\
\text { Maximum pip in all trades. }\end{array}$ \\
total_profit & $\begin{array}{l}\text { Earned profit from all trades. } \\
\text { avg_time_lag } \\
\text { Average time lag between news announcements } \\
\text { and trade open time. }\end{array}$ \\
news_ref_pct & $\begin{array}{l}\text { Percentages of trades operated before and after the news } \\
\text { announcement among all the trades with the currency } \\
\text { mentioned in that news announcement. }\end{array}$ \\
\hline \hline
\end{tabular}

avg_time_lag and news_ref_pct. For a news announcement, at first we detect the concerned currencies. Then we search for the trades on those currencies placed before and after the news announcement. Currently, we limit the time window to one hour, ranging from thirty minutes before to thirty minutes after the news announcement.

For each factor, before estimating a trader's performance level we standardize the factor value by comparing the trader with other traders. This makes our method become context aware. We compare the factors of a target trader $u$ with those of the other traders $U$ to standardize each factor value. Intuitively, we can say that high pip, win_rate, total_profit, news_ref_pct and low avg_time_lag indicate high performance level. The performance level of trader $u, \operatorname{per}(u, U)$ is defined as follows:

$$
\begin{aligned}
& \operatorname{per}(u, U)=p_{1} * z(\text { win_rate }, u, U) \\
& +p_{2} * z\left(a v g \_p i p, u, U\right)+p_{3} * z\left(\max \_p i p, u, U\right) \\
& +p_{4} * z(\text { total_profit, } u, U)+p_{5} * z\left(a v g \_t i m e \_l a g, u, U\right) \\
& +p_{6} * z\left(n e w s \_r e f \_p c t, u, U\right) \\
& z(x, u, U)=\frac{(x(u)-\operatorname{avg}(x(U))) * 10}{\operatorname{std}(x(U))} \\
& \begin{array}{r}
z\left(\max \_p i p, u, U\right)= \\
\left(\frac{1}{m d\left(m a x \_p i p(u), \text { max_pip }(U)\right)}+1\right) * \\
\frac{\left(m a x \_p i p(u)-a v g\left(\max \_p i p(U)\right)\right) * 10}{\text { std }(\text { max_pip }(U))}
\end{array} \\
& \text { md }(\text { max_pip }(u), \text { max_pip }(U))= \\
& \frac{\left.\mid \max \_p i p(u)-\operatorname{avg}\left(\max \_p i p(U)\right)\right) \mid}{s t d\left(\max \_p i p(U)\right)}
\end{aligned}
$$

where $p_{i}$ is the weight parameter; $z()$ denotes the standardization of each factor; $u$ and $U$ are used as respective arguments of function $x()$, while $x$ can be one of win_rate, avg_pips, total_profit, avg_time_lag, and news_ref_pct; $\operatorname{avg}()$ denotes average function; std() denotes standard deviation function; and $m d()$ denotes Mahalanobis distance, which is used to calculate how far away the maximum value is from
TABLE II

FACTORS USED FOR RISK ESTIMATION

\begin{tabular}{ll}
\hline \hline Drawdown & $\begin{array}{l}\text { Measure of the decline from the open- } \\
\text { ing price during a certain trade. } \\
\text { Trade duration from open (buying) to } \\
\text { close (selling). } \\
\text { Duration }\end{array}$ \\
Maximum Drawdown $\left(d d_{\max }\right)$ & $\begin{array}{l}\text { Maximum drawdown in all trades. } \\
\text { Average drawdown in all trades. }\end{array}$ \\
Maxage Drawdown $\left(d d_{a v g}\right)$ & Maximum duration in all trades. \\
Average Duration $\left(d\right.$ dur $\left._{\text {avg }}\right)$ & Average duration in all trades. \\
\hline \hline
\end{tabular}

the mean value and whether it is a meaningful value that affects the factor, because maximum values may appear only once in most cases.

\section{B. Risk Estimation by Comparing with Other Traders}

We estimate the risk level of traders in a way similar to that we use to estimate their performance: by using factors obtained from traders' profiles. The details of these factors are shown in Table II. In the same way as used to estimate performance, we compare the factors for trader $u$ with those of the other traders $U$ to standardize each factor value.

Intuitively, we can say that small drawdown and short trade duration indicate low risk level. The risk level of trader $u$, $\operatorname{risk}(u, U)$ is defined as follows:

$$
\begin{array}{r}
\operatorname{risk}(u, U)=r_{1} * z\left(d d_{\max }, u, U\right)+r_{2} * z\left(d d_{\text {avg }}, u, U\right) \\
+r_{3} * z\left(d u r_{\max }, u, U\right)+r_{4} * z\left(d u r_{\text {avg }}, u, U\right) \\
z(x, u, U)=\left(\frac{1}{\operatorname{md}(x(u), x(U))}+1\right) \\
* \frac{(x(u)-\operatorname{avg}(x(U))) * 10}{\operatorname{std}(x(U))} \\
\operatorname{md}(x(u), x(U))=\frac{|x(u)-\operatorname{avg}(x(U))|}{\operatorname{std}(x(U))} \\
z(y, u, U)=\frac{(y(u)-\operatorname{avg}(y(U))) * 10}{\operatorname{std}(y(U))}
\end{array}
$$

where $r_{i}$ is the weight parameter, $u$ and $U$ are used as respective arguments of function $x(), x$ stands for $d d_{\text {max }}$ or $d u r_{\max }$, and function $y$ stands for $d d_{a v g}$ or $d u r_{a v g}$.

\section{Consistency Estimation by Comparing with Historical} Trades

Some users may want to search for expert traders with relative consistent performance to follow. Following such experts in mirror trades is a reasonable way to make profit in social trading services. Therefore, this raises a challenge to estimate the consistency of a trader. Currently, we use the trade records of traders to estimate their volatility. We calculate the standard deviation of the factors shown in Table III. Intuitively, we can say that small deviation indicates high consistency. We introduce two new factors: the number of currencies and the number of trades. These are based on an observation from an investigation on the consistency of traders[9], i.e., consistent 
TABLE III

FACTORS USED FOR CONSISTENCY ESTIMATION

\begin{tabular}{|c|c|}
\hline SD of pip $\left(s t d_{\text {pip }}\right)$ & $\begin{array}{l}\text { Standard deviation of a trader's } \\
\text { pips in a certain duration. }\end{array}$ \\
\hline$S D$ of drawdown $\left(s_{d d}\right)$ & $\begin{array}{l}\text { Standard deviation of trade draw- } \\
\text { downs in a certain duration. }\end{array}$ \\
\hline$S D$ of duration $\left(s t d_{d u r}\right)$ & $\begin{array}{l}\text { Standard deviation of trade dura- } \\
\text { tions in a certain duration. }\end{array}$ \\
\hline $\begin{array}{l}\text { SD of number of currencies con- } \\
\left.\text { currently operated (std } d_{\text {cur }}\right)\end{array}$ & $\begin{array}{l}\text { Standard deviation of number of } \\
\text { currencies which are traded con- } \\
\text { currently in a certain duration. }\end{array}$ \\
\hline$S D$ of number of trades $\left(s t d_{\text {trad }}\right)$ & $\begin{array}{l}\text { Standard deviation of the number } \\
\text { of trades operated simultaneously. }\end{array}$ \\
\hline
\end{tabular}

traders focus on a small number of currencies and trades. The formula for calculating the consistency of trader $u$ in duration $\tau, \operatorname{con}(u, \tau)$, is given below. We subtract $s t d_{\text {cur }}$ and $s t d_{\text {simul }}$ because those values represent how many trades are operated at the same time. We think that this is the reason for the lower concentration of traders.

$$
\begin{array}{r}
\operatorname{con}(u, \tau, U)=c_{1} * z\left(s t d_{\text {pip }}(u, \tau), U\right) \\
+c_{2} * z\left(s t d_{d d}(u, \tau), U\right)+c_{3} * z\left(\operatorname{std}_{d u r}(u, \tau), U\right) \\
-c_{4} * z\left(s t d_{c u r}(u, \tau), U\right)-c_{5} * z\left(s t d_{\text {simul }}(u, \tau), U\right) \\
z(x(u, \tau), U)=\frac{(x(u, \tau)-\operatorname{avg}(x(U, \tau))) * 10}{\operatorname{std}(x(U, \tau))}
\end{array}
$$

Here, $c_{i}$ is the weight parameter; $u$ indicates trader $\mathrm{u}$; $U$ indicates all traders; $u$ and $U$ are used as respective arguments of function $x()$, while $x$ can be one of $s t d_{p i p}, s t d_{d d}, s t d_{d u r}$, $s t d_{c u r}$, and $s t d_{\text {simul }} ; \tau$ denotes the retrospective period of each trader from now to his/her first trade. Currently, we limit the retrospective scope to a maximum of five years.

\section{EXPERIMENTS}

We carried out experiments to evaluate the three measures (performance, risk, and consistency) by applying them to rank traders. We also evaluated the proposed W2F system on the basis of a user survey.

\section{A. Evaluation of Proposed Measures}

We compared the ranking results on the basis of the proposed three measures and the original one available on the Web site of Zulutrade. We compared these two kinds of ranking results from the viewpoint of the ranking accuracy by using nDCG (normalized Discounted Cumulative Gain). Furthermore, we will discuss an integrated way to rank traders by combining the three measures.

The $\mathrm{nDCG}$ score regarding a ranking of the top $p$ is defined as follows:

$$
n D C G_{p}=\frac{D C G_{p}}{I D C G_{p}}
$$

where $D C G_{p}$ is a weighted score. If we let the score of the $i$-th expert trader be $r e l_{i}, D C G_{p}$ is expressed as

$$
D C G_{p}=r e l_{1}+\sum_{i=1}^{p} \frac{r e l_{i}}{\log _{2} i}
$$

where $I D C G_{p}$ is a value that applies the ideal ranking arranged in descending order of scores to expression (Formula 12). The ranking achieved approaches the ideal as the $n D C G$ value becomes higher.

1) Dataset: We downloaded the profiles (including trading history) of 100 traders top-ranked on Zulutrade on December 31, 2014. Also, from ForexFactory we downloaded necessary news/event data of eight years consisting of the event date, time of announcement, title, target currencies, impact level of the market, and so on. These profiles and news data are stored and managed by using MySQL in our current implementation.

2) Evaluation on Ranking Accuracy: At first, we used only one of the three measures (performance, risk, and consistency) to rank the 100 traders. In addition, because by using our W2F system a user may find expert traders by considering all three measures, we calculated the nDCG score in the case of ranking traders by combining the three measures as

$$
\begin{array}{r}
\operatorname{score}_{\text {prc }}(u, U)=w_{1} * \operatorname{per}(u, U) \\
+w_{2} * \operatorname{risk}(u, U)+w_{3} * \operatorname{con}(u, \tau, U)
\end{array}
$$

where $w_{1}, w_{2}$ and $w_{3}$ are weight parameters.

We asked four evaluators to score the given 100 traders using a five-grade evaluation method to get ideal rankings. We asked them to check the profiles on Zulutrade to perform these tasks. We used the results returned by the evaluator who is most familiar with Forex to tune the parameters. The nDCG scores with varied parameters are shown in Table IV, V, VI, and VII. We found that assigning $(1,2,2,2,2,1)$ to $\left(p_{1}, p_{2}, p_{3}, p_{4}, p_{5}, p_{6}\right)$ respectively is a set of proper parameters that can achieve reasonable results, when we only considered the performance (Table IV). Also, we found out assigning $(10,3,3,2)$ to $\left(r_{1}, r_{2}, r_{3}, r_{4}\right)$ respectively constitutes a set of proper parameters that achieves the best $n D C G_{30}$ value 0.99 when we only considered the risk (Table V). The weight parameters $(1.5,1,1,10,1)$ achieve the best accuracy in ranking

\begin{tabular}{|c|c|c|c|c|c|c|c|c|c|}
\hline \multirow{2}{*}{ Method } & \multicolumn{6}{|c|}{ Parameters } & \multicolumn{3}{|c|}{$n D C G$} \\
\hline & $p_{1}$ & $p_{2}$ & $p_{3}$ & $p_{4}$ & $p_{5}$ & $p_{6}$ & top10 & top20 & top30 \\
\hline \multirow{4}{*}{$\begin{array}{l}\text { P(Perfo- } \\
\text { rmance) }\end{array}$} & 1.5 & 1.5 & 1 & 1.5 & 1.5 & 1 & 0.98 & 0.97 & 0.98 \\
\hline & 1 & 2 & 2 & 2 & 2 & 1 & 1 & 0.98 & 0.98 \\
\hline & 1 & 2 & 1 & 2 & 2 & 1 & 1 & 0.98 & 0.99 \\
\hline & 1 & 1 & 1 & 1 & 1 & 1 & 0.97 & 0.97 & 0.95 \\
\hline Zulutrade & - & & & & & & 0.66 & 0.67 & 0.71 \\
\hline
\end{tabular}
traders from the aspect of consistency (Table VI). Finally, we found that assigning $(1,2,6)$ to $\left(w_{1}, w_{2}, w_{3}\right)$ respectively constitutes a set of proper parameters that can achieve reasonable results when we considered all three measures (Table VII). We used these parameter values for further processing.

TABLE IV

Results for Tuning Parameters: Performance

The results returned by the other three evaluators were used for evaluating the proposed measures. We averaged the $\mathrm{nDCG}$ scores calculated on the basis of the results given by the three evaluators. As shown in Table VIII, the proposed measures of risk and consistency outperformed the original ones of Zulutrade. However, the results for performance were not good 
TABLE V

RESUlts FOR TUNING PARAMETERS: RISK

\begin{tabular}{l|llll|lll}
\hline \hline \multirow{2}{*}{ Method } & \multicolumn{3}{|l|}{ Parameters } & \multicolumn{3}{l}{$n D C G$} & \\
\cline { 2 - 8 } & $p_{1}$ & $p_{2}$ & $p_{3}$ & $p_{4}$ & top10 & top20 & top30 \\
\hline \multirow{3}{*}{ R(Risk) } & $\mathbf{1 0}$ & $\mathbf{3}$ & $\mathbf{3}$ & $\mathbf{2}$ & $\mathbf{0 . 9 8}$ & $\mathbf{0 . 9 7}$ & $\mathbf{0 . 9 9}$ \\
& 10 & 1 & 3 & 2 & 1 & 0.96 & 0.98 \\
& 20 & 1 & 3 & 2 & 0.94 & 0.94 & 0.94 \\
& 20 & 3 & 3 & 3 & 0.97 & 0.95 & 0.97 \\
\hline Zulutrade & - & & & 0.63 & 0.65 & 0.72 \\
\hline \hline
\end{tabular}

TABLE VI

RESUlts FOR TUNING PARAMETERs: CONSISTENCY

\begin{tabular}{l|lllll|lll}
\hline \hline \multirow{2}{*}{ Method } & \multicolumn{3}{|l|}{ Parameters } & & & & & \\
& $p_{1}$ & $p_{2}$ & $p_{3}$ & $p_{4}$ & $p_{5}$ & top10 & top20 & top30 \\
\hline \multirow{2}{*}{ C(Consis- } & 1 & 1 & 1 & 10 & 1 & 1 & 0.98 & 0.98 \\
tency) $)$ & $\mathbf{1 . 5}$ & $\mathbf{1}$ & $\mathbf{1}$ & $\mathbf{1 0}$ & $\mathbf{1}$ & $\mathbf{1}$ & $\mathbf{1}$ & $\mathbf{1}$ \\
& 1.5 & 1.5 & 1 & 10 & 1 & 1 & 0.98 & 0.99 \\
& 1.5 & 1.5 & 1 & 1 & 1 & 0.97 & 0.96 & 0.97 \\
\hline Zulutrade & - & & & & & 0.66 & 0.67 & 0.71 \\
\hline \hline
\end{tabular}

as we expected. One of the major reasons for this is that some evaluators only considered total profit to evaluate the traders' performance levels. The results also indicate that considering all three measures can achieve reasonable results.

3) User Study of W2F: We also carried out a survey targeting the four evaluators who participated in the above experiments. We asked them to use both W2F and Zulutrade and then compare them. As shown in Table IX, we asked two questions to evaluate the usefulness of W2F. Three of the four evaluators said that our W2F system was more helpful for understanding the characteristics of traders and then finding expert traders. These results reveal that, although the user interface needs further improvement and more support of user interaction is necessary, our system can help users discover expert traders.

\section{CONCLUSION}

In the first attempt at discovering expert traders on social trading services, we have proposed three measures (performance, risk, and consistency) and developed a system we call W2F (Whom to Follow), to help users select expert traders to follow. In contrast to the current services, the notable features of our method of estimating traders include 1) taking the consistency of trading behaviors into consideration and 2) providing a relative ranking method with which to compare other traders. We also carried out experiments to validate the proposed measures for estimating traders from the viewpoint of ranking accuracy. A user study on W2F was also performed. The experimental results reveal that the proposed measures and $\mathrm{W} 2 \mathrm{~F}$ are useful in helping users discover expert traders to follow.

In the near future, we plan to carry out further experiments to evaluate and improve W2F. Applying a deep learning method to aid in discovering expert traders is also in our future plans. In addition, we will attempt to improve the user interface of W2F and introduce a relevance feedback function into it.
TABLE VII

Results for Tuning Parameters: Considering Three Aspects

\begin{tabular}{l|l|lll}
\hline \hline \multirow{2}{*}{ Method } & \multirow{2}{*}{ Weight } & \multicolumn{3}{|c}{$n D C G$} \\
\cline { 2 - 5 } & $\left(w_{i}, w_{i}, w_{i}\right), i=1,2,3$ & top10 & top20 & top30 \\
\hline \multirow{5}{*}{ PRC } & $(1,2,2)$ & 0.96 & 0.93 & 0.91 \\
& $(\mathbf{1 , 2 , 6 )}$ & $\mathbf{0 . 9 6}$ & $\mathbf{0 . 9 3}$ & $\mathbf{0 . 9 1}$ \\
& $(1,1,2)$ & 0.92 & 0.92 & 0.91 \\
& $(2,3,1)$ & 0.92 & 0.92 & 0.91 \\
\hline
\end{tabular}

TABLE VIII

RESULTS OF NDCG (WHERE PRC DENOTES THE COMBINATION OF PERFORMANCE, RISK, AND CONSISTENCY

\begin{tabular}{ll|llll}
\hline \hline \multicolumn{2}{c|}{ Method } & Performance & Risk & Consistency & PRC \\
\hline \multirow{3}{*}{ W2F } & $n D C G_{10}$ & 0.76 & 0.66 & 0.78 & 0.84 \\
& $n D C G_{20}$ & $\mathbf{0 . 7 5}$ & $\mathbf{0 . 6 4}$ & $\mathbf{0 . 7 6}$ & $\mathbf{0 . 8 5}$ \\
& $n D C G_{30}$ & 0.77 & 0.63 & 0.77 & 0.82 \\
\hline \multirow{3}{*}{ Zulutrade } & $n D C G_{10}$ & 0.81 & 0.58 & 0.70 & 0.76 \\
& $n D C G_{20}$ & 0.80 & 0.56 & 0.67 & 0.77 \\
& $n D C G_{30}$ & 0.82 & 0.58 & 0.71 & 0.78 \\
\hline \hline
\end{tabular}

TABLE IX

Number of EVAluators ANSWERING "Yes"In SURVEY ON W2F AND ZULUTRADE

\begin{tabular}{l|cc}
\hline \hline \multicolumn{1}{c|}{ Questionnaire } & W2F & Zulutrade \\
\hline $\begin{array}{l}\text { Which system enabled you to more easily } \\
\text { recognize traders' trading characteristics? }\end{array}$ & 3 & 1 \\
\hline $\begin{array}{l}\text { Which system do you think makes it easier } \\
\text { to find the trader you want? }\end{array}$ & 3 & 1 \\
\hline \hline
\end{tabular}

\section{ACKNOWLEDGMENT}

This work is partly supported by KAKENHI(No.25700033) and SCAT Research Funding.

\section{REFERENCES}

[1] Luc Bauwens, Walid Ben Omrane, Pierre Giot: News announcements, market activity and volatility in the euro/dollar foreign exchange market. Journal of International Money and Finance 24(7) 2005: 1108-1125

[2] Wei Pan, Yaniv Altshuler, Alex Pentland: Decoding Social Influence and the Wisdom of the Crowd in Financial Trading Network. SocialCom/PASSAT 2012: 203-209

[3] Craig Macdonald, David Hannah, Iadh Ounis: High Quality Expertise Evidence for Expert Search. ECIR 2008: 283-295

[4] Ioannis Konstas, Vassilios Stathopoulos, Joemon M. Jose: On social networks and collaborative recommendation. SIGIR 2009: 195-202

[5] Abeer El-Korany: Integrated Expert Recommendation Model for Online Communities. International Journal of Web \& Semantic Technology 4(4) 2013: 19-29

[6] Yang-Yu Liu, Jose C. Nacher, Tomoshiro Ochiai, Mauro Martino, Yaniv Altshuler: Prospect Theory for Online Financial Trading. APS March Meeting 2014: e109458

[7] Gyozo Gidofalvi: Using news articles to predict stock price movements. 2001: 1-9

[8] Social Trading(Wikipedia): http://en.wikipedia.org/wiki/Social_trading

[9] SocialTradingGuru: http://socialtradingguru.com

[10] Zulutrade: http://www.zulutrade.com

[11] Ayondo: http://www.ayondo.com

[12] eToro: http://www.etoro.com

[13] Stackoverflow: http://stackoverflow.com

[14] ForexFactory: http://www.forexfactory.com 